${ }^{1}$ Hospital Base de Puerto Montt. Puerto Montt, Chile. 2Universidad San Sebastián, Puerto Montt. Chile. 3Universidad de La Frontera. Temuco, Chile. ${ }^{4}$ Departamento de Cirugía, Traumatología y Anestesia. Universidad de La Frontera. Temuco, Chile.

${ }^{5}$ Hospital Doctor Hernán Henríquez Aravena. Temuco, Chile.

aBecado Anestesiología y Reanimación, Universidad San Sebastian Puerto Montt, Chile.

bInterno de Medicina, Universidad de La Frontera, Temuco, Chile. 'MSc, MBA.

Recibido el 5 de junio de 2018, aceptado el 4 de diciembre de 2018.

Correspondencia a: Waldo Merino Urrutia Los Pablos 2860 casa 45, Temuco, Chile.

Wamerino@gmail.com

\section{Huella de carbono en el Hospital Base de Puerto Montt}

\author{
MARCO BALKENHOL ${ }^{1}$, ALEJANDRO CASTILLO ${ }^{2, \mathrm{a}}$, MICHAEL SOTO ${ }^{3, \mathrm{~b}}$, \\ MATÍAS FEIJOO ${ }^{3, \mathrm{~b}}$, WALDO MERINO ${ }^{4,5, \mathrm{c}}$
}

\section{Measurement of the carbon footprint at a regional hospital}

Background: Measuring the carbon footprint (CF) makes it possible to estimate the contribution of clinical activity to global warming. Aim: To measure the emitter components of $\mathrm{CO} 2$ equivalents (CO2e) at the Hospital Base, Puerto Montt (HBPM). Material and methods: Descriptive study with data collected retrospectively between January and December 2016 from the HBPM database. The data analyzes direct and indirect emissions as well as other indirect emissions beyond the organizational limits. Results: Of the 9,660.3 tons of CO2e emitted by the HBPM in 2016, 46\% were derived from consumption of electricity, 29\% derived from the generation of residues, and $10 \%$ from clinical gas consumption, of which Sevoflurane was the greatest contributor. Conclusions: Clinical gases are a significant source of CO2e emissions. Sevoflurane alone is in fourth place in CO2e emissions at the HBPM. Estimating the CF produced by HBPM is the first step in the discussion of measures to reduce the environmental impact of our activity.

(Rev Med Chile 2018; 146: 1384-1389)

Key words: Anesthetics; Carbon Footprint; Climate Change; Global Warming.

\footnotetext{
$\tau$
} a evidencia sugiere que la actividad humana está asociada al calentamiento global y este a su vez con el cambio climático. Este es un fenómeno establecido que amenaza la salud de las personas y que se presenta como un desafío urgente por mitigar a través de la disminución en la producción de los llamados gases con efecto invernadero $(\mathrm{GEI})^{1-3}$.

Los GEI son un conjunto de compuestos atmosféricos, algunos de ellos presentes de forma natural como el $\mathrm{CO} 2$ y otros introducidos artificialmente por la actividad humana como los hidrofluorocarburos. Estos absorben y re-emiten hacia la corteza la radiación infrarroja impidiendo que parte del calor del sol sea reflejado, permaneciendo en la tierra. Se estima que su drástico aumento, en las últimas décadas, ha colaborado en el incremento de la temperatura en la superficie del planeta $^{2,4}$. La huella de carbono (HC) es un térmi- no usado para describir la cantidad de GEI que son liberados a la atmósfera directa o indirectamente como consecuencia de una actividad humana ${ }^{5}$.

Paradójicamente, el sector salud es responsable de un porcentaje importante de la HC en países desarrollados como Estados Unidos, donde las emisiones alcanzan entre 8 y $10 \%$ de la producción total de GEI o en el Reino Unido donde genera $25 \%$ de la $\mathrm{HC}$ del sector público ${ }^{3,6}$. MacNeill AJ et al, han descrito recientemente la $\mathrm{HC}$ de tres hospitales docentes en tres servicios de salud de Canadá, Estados Unidos y el Reino Unido, llama la atención el significativo aporte de la actividad quirúrgica y especialmente de los anestésicos halogenados en la emisión de $\mathrm{GEI}^{6}$. En nuestro país encontramos escasas publicaciones que den cuenta del impacto que genera la actividad clínica de servicios y hospitales, y de la contribución de los gases utilizados en la práctica anestésica. 
Así, pretendemos cuantificar y describir los componentes emisores de GEI en el Hospital Base de Puerto Montt (HBPM) para aportar con información relevante en la discusión del impacto que tiene nuestra actividad clínica en el cambio climático y en la salud de las personas.

\section{Materiales y Métodos}

Este es un estudio descriptivo, con información obtenida retrospectivamente entre enero y diciembre de 2016, desde la base de datos del HBPM. Se obtuvo antecedentes de consumo y adquisición de energía eléctrica, combustibles, agua, papel (formato para impresora y de uso higiénico), gases clínicos y generación de residuos, entre otros.

La información fue tabulada en hojas de cálculo de Microsoft Excel $^{\odot}$ y almacenada en la plataforma Google Drive, obtenida y organizada de acuerdo al "Protocolo de Gases de Efecto Invernadero" que establece un análisis basándose en tres alcances ${ }^{7}$ :

1) Emisiones directas: Como el consumo de combustible fósil o procesos de emisión específicos.

2) Emisiones indirectas: asociadas a la adquisición y consumo de la energía eléctrica.

3) Otras emisiones indirectas producidas por actividades o procesos que están fuera de los límites organizacionales ${ }^{8}$.

En el primer alcance se obtuvo el consumo de combustible fósil asociado a fuentes fijas (calderas y grupo electrógeno) y fuentes móviles (vehículos del servicio) correspondiendo a gas licuado de petróleo (GLP), diésel y también los gases clínicos.

Dentro de la tabla no se incluyeron los datos derivados del consumo de biomasa en calderas, ya que su aporte a la HC se considera neutro, aunque son mencionados. Tampoco se consideraron las emisiones por fugas, las asociadas al transporte de personal ni las derivadas de faenas de construcción.

En el segundo alcance se incluyeron los datos de consumo anual de energía eléctrica del HBPM, obtenidas a través del resumen de facturas de suministro eléctrico.

En el tercer alcance se incluyeron los datos de consumo de agua potable, consumo de papel anual (en $\mathrm{Kg}$ de papel utilizado en labores administrativas e higiénicas) y generación anual de residuos en su totalidad (los que no fueron diferenciados). El total de los datos fueron tabulados y multiplicados por sus respectivos factores de conversión para obtener la $\mathrm{HC}$ reflejada en toneladas equivalentes de $\mathrm{CO} 2(\mathrm{CO} 2 \mathrm{e})^{9,10}$, unidad que puede ser definida como la cantidad de $\mathrm{CO} 2 \mathrm{e}$ emitido que provocaría la misma intensidad radiante que una mezcla determinada de $\mathrm{CO} 2 \mathrm{y} / \mathrm{u}$ otros $\mathrm{GEI}^{11,12}$.

\section{Resultados}

En la Tabla 1 se presentan las emisiones generadas por los insumos utilizados en los procesos

Tabla 1. Resumen de variables y producción de CO2e para el HBPM

\begin{tabular}{|c|c|c|c|}
\hline Variables & $\begin{array}{c}\text { Consumo anual } \\
(2016)\end{array}$ & $\begin{array}{l}\text { Factor de emisión } \\
\text { (kg CO2 eq/ud) }\end{array}$ & TonEq CO2 \\
\hline Diésel (litros) & $310.924,00$ & 2,68 & 833,30 \\
\hline GLP (kg) & $36.284,40$ & 3,03 & 109,90 \\
\hline Electricidad (kWh) & $11.422 .980,00$ & 0,397 & $4.534,90$ \\
\hline Agua (litros) & $140.257,00$ & 0,788 & 110,50 \\
\hline Papel común (kg) & $89.806,80$ & 3,00 & 269,40 \\
\hline Óxido nitroso (kg) & 381,00 & 289,00 & 110,10 \\
\hline Sevoflorano (kg) & 382,10 & $1.980,00$ & 756,30 \\
\hline Desflorano (kg) & 22,60 & $3.714,00$ & 83,90 \\
\hline Dióxido de carbono (kg) & $1.258,00$ & 1,00 & 1,30 \\
\hline Generación de residuos (kg) & $2.522 .724,20$ & 1,13 & $2.850,70$ \\
\hline Pellet madera (kg) & $2.134 .800,00$ & 0 & 0 \\
\hline
\end{tabular}

Total en toneladas equivalentes de $\mathrm{CO}_{2} . \mathrm{Kg} \mathrm{CO} 2 \mathrm{eq} / \mathrm{ud}$ : Kilogramos de $\mathrm{CO} 2$ equivalentes por unidad de medida de variable mencionada. TonEq CO2: Toneladas equivalentes de $\mathrm{CO} 2$ producidas por cada variable. 
del HBPM durante el año 2016 expresados en toneladas de $\mathrm{CO} 2 \mathrm{e}$ de acuerdo a las conversiones señaladas en la literatura, ${ }^{9,10}$.

Dentro de la base de datos consultada, se obtuvo los valores de consumo anual de las variables correspondientes a los alcances 1,2 y 3 , se incluyen los gases anestésicos en el primer alcance. Estas cantidades de recursos consumidos y residuos generados se multiplican por factores de emisión de CO2e correspondientes ${ }^{9,10}$, lo que da como resultado la emisión de toneladas de $\mathrm{CO} 2$ e en un año (Tabla 1). Se incluye además el consumo anual de biomasa pellet, que corresponde a $2.134,8$ toneladas. Si bien su factor de emisión de CO2e es neutro, aporta con un equivalente energético de 10.428.241 kWh/año, equiparando en $91,3 \%$ al consumo eléctrico.

De las 9.660,3 toneladas de CO2e emitidas por el HBPM durante el año 2016, destaca 46\% correspondiente al consumo eléctrico, seguido por la generación de residuos con $29 \%$ y en un tercer lugar se encuentra el consumo de gases clínicos, con $10 \%$ (Figuras 1 y 2). Dentro de los gases clínicos, el principal productor de $\mathrm{CO} 2 \mathrm{e}$, corresponde al halogenado sevofluorano (Figura $3)$.

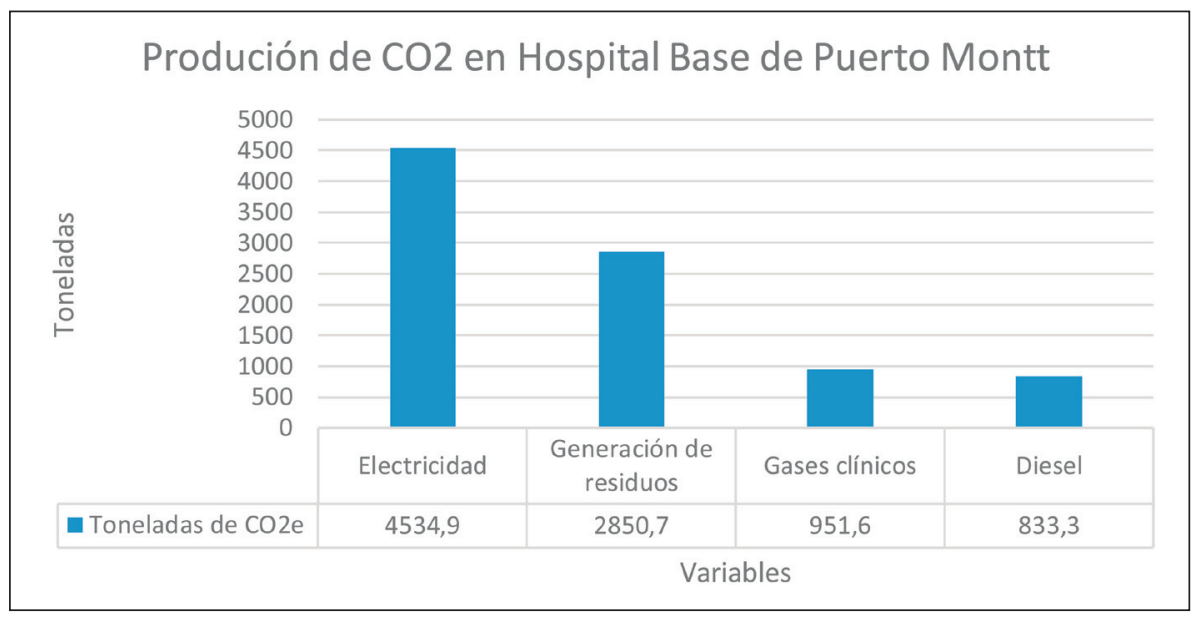

Figura 1. Emisión de CO2e en Hospital Base de Puerto Montt. Los gases clínicos representan el tercer lugar en producción de $\mathrm{CO} 2$ e.

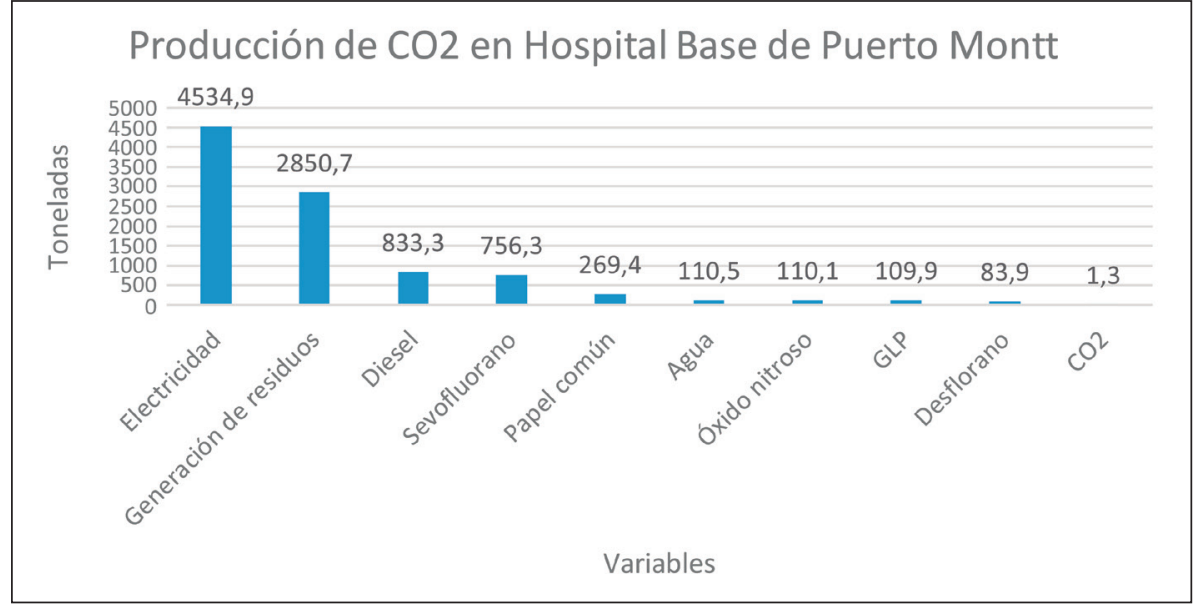

Figura 2. Emisión de CO2e en HBPM. El Sevofluorano representa el cuarto lugar en producción de $\mathrm{CO} 2 \mathrm{e}$. 


\section{APORTE DE CO2 EMITIDO DE LOS GASES ANESTÉSICOS USADOS EN HBPM}

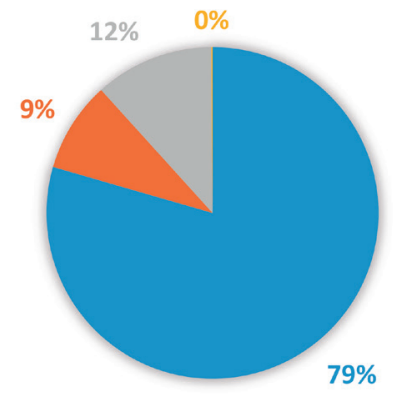

- Sevofluorano

- Desflorano

- Óxido nitroso

$\mathrm{CO} 2$

Figura 3. Proporción de CO2e emitido por gases anestésicos en HBPM. El Sevofluorano en color azul representa aproximadamente el $80 \%$ de la proporción de CO2e emitido.

\section{Discusión}

Ante la llegada del cambio climático y sus consecuencias, se proyecta un incremento en la mortalidad anual y mayores costos en salud. Se predice un aumento de las enfermedades infecciosas y cardiovasculares asociados a los cambios ambientales generados por las variaciones de la temperatura, así como un aumento en las catástrofes naturales (como sequías o inundaciones) con sus respectivas consecuencias a nivel sanitario ${ }^{1}$.

A pesar de que la OMS ha resaltado la importancia del cambio climático y su impacto en la salud pública, es la industria del cuidado de la salud una de las principales emisoras de $\mathrm{CO} 2 \mathrm{e}^{13}$, principalmente por el uso de energía en sus edificios, así como distintos procesos realizados a diario (transporte de pacientes, generación de residuos, etc.) o la liberación de gases anestésicos y medicinales al ambiente ${ }^{14}$. Entre estos gases podemos mencionar los gases halogenados, de los cuales se han estudiado halotano, isofluorano, sevofluorano y desflurano, los que tienen un Potencial de Calentamiento Global considerablemente más alto que el del $\mathrm{CO} 2^{15}$.

De la misma forma el $\mathrm{N} 2 \mathrm{O}$, ampliamente usado en anestesia, juega un rol como GEI y así también en la degradación del ozono atmosférico ${ }^{4}$. Se estima que al utilizarlo como mezcla N2O/ O2 Puede aumentar hasta 65\% las emisiones de GEI para isofluorano y $900 \%$ para sevofluorano (al usarse en una proporción 60/40 N2O/O2), en comparación a la mezcla aire/O2 $2^{13,15}$.

Describimos a los gases anestésicos como generadores importantes de CO2e. Son la tercera fuente de emisión en el HBPM, alcanzando $10 \%$ del total. Destaca el sevofluorano que por sí solo alcanza el $4^{\circ}$ lugar, apenas superado por el diésel. El amplio uso que se le da al sevofluorano contrasta con el escaso uso de desfluorano, que a pesar de tener un mayor factor de emisión su aporte es menor. El isofluorano no está presente en el arsenal farmacológico. Así, La emisión de CO2e a propósito de la práctica anestésica en el HBPM está principalmente sostenido por el uso de sevofluorano.

Macneill et al. ${ }^{6}$ estimaron la HC en 3 pabellones quirúrgicos de Inglaterra, Estados Unidos y Canadá. El CO2e generado por los anestésicos en los centros norteamericanos superó el 50\% del total de las emisiones de ambos hospitales, mientras que en Inglaterra sólo alcanza 4\%, debido al uso de otras técnicas anestésicas como la anestesia total intravenosa y anestesia regional en lugar de gases. Esto es un claro ejemplo de cómo la técnica anestésica determina una diferencia importante en la HC. Sorprendentemente el total de CO2e emitido en el mismo hospital es mayor que en los otros centros, ya que la energía eléctrica que utiliza es generada por carbón y gas natural.

Debido al aporte de la anestesia en la emisión de GEI, es que debemos plantearnos el desafío de hallar nuevas tecnologías que disminuyan las emisiones de estos gases al ambiente, como el desarrollo de fármacos que generen un menor impacto ambiental ${ }^{4,15}$. Jodi Sherman et al. nos señalan que una alternativa a los gases es el uso de agentes anestésicos endovenosos como el propofol. La emisión de GEI de este fármaco se encuentra fundamentalmente en la jeringa con la que se administra y la electricidad utilizada por la bomba de infusión, generando un impacto ambiental considerablemente menor ${ }^{13}$. Este estudio no especifica la $\mathrm{HC}$ generada en la manufactura de estos fármacos, sólo realiza una estimación de la misma.

Entre las recomendaciones que ayudan a disminuir el impacto ambiental de la práctica anestésica se encuentra el uso de bajos flujos de gases frescos (FGF). Esta técnica consiste en administrar FGF inferior a la ventilación minuto del paciente, así mediante un sistema de reinhalación, cerrado o 
semicerrado, el gas anestésico vuelve a incorporarse. La utilización de circuitos cerrados y de flujos bajos de gases pueden reducir el volumen consumido de gases anestésicos inhalatorios en $80 \%$ a $90 \%{ }^{14}$. La utilización de estas técnicas signficarían una gran reducción de la cantidad de $\mathrm{CO} 2$ e que se arroja a la atmósfera, pasando de 17,64 toneladas de CO2e por año a un rango entre 1,73 a $3,53^{16}$. Otras medidas, como el relleno hermético de los vaporizadores, controlar fugas en los circuitos, el uso de mascarillas y tubos endotraqueales con cuff que sellen completamente la vía aérea y el uso de la anestesia regional son recomendadas. Actualmente se están desarrollando sistemas de reciclaje de gases anestésicos y explorando el uso del gas xenón. Entre las características de este gas destaca lograr analgesia profunda, neuroprotección, estabilidad hemodinámica y carecer de efectos ecotóxicos conocidos. Sin embargo, su uso se ve limitado por el alto consumo de energía que requiere su producción $(220 \mathrm{~W} / \mathrm{h}$ para $1 \mathrm{~L}$ de xenón $)^{13,17}$. Las opciones de las que actualmente disponemos para disminuir la huella de carbono han sido revisadas por McGain et al. ${ }^{18} \mathrm{y}$ se resumen en la Tabla 2.
Tras entender los efectos del calentamiento global y el rol de los centros sanitarios en la generación de GEI, entendemos como un desafío ineludible la elaboración de medidas destinadas a disminuir nuestro aporte en la HC. Medidas como disminuir el consumo de combustibles, el uso de fuentes renovables para la generación de electricidad, así como el manejo eficiente de residuos contribuyen a disminuir la $\mathrm{HC}$ producida en nuestros hospitales. Es necesario impulsar políticas ambientales como la construcción de centros sanitarios eficientes del punto de vista energético y establecer en las licitaciones públicas, como requisito, un informe del impacto en la $\mathrm{HC}$ que tienen los insumos ofertados. En el que hacer clínico observar el uso racional y riguroso de los recursos, considerando las fuentes de $\mathrm{CO} 2$ equivalente no solo por el impacto en la HC generado en la práctica clínica sino también en su manufactura. Es así como el NHS (national health service) de Reino Unido estableció que "el manejo del carbono es un tema cada vez más importante para todas las organizaciones. Tomar en serio la sustentabilidad y las emisiones de carbono es una parte integral de un servicio de salud de alta calidad"5.

Tabla 2. Recomendaciones para disminuir la huella de carbono en pabellón

\begin{tabular}{|c|c|}
\hline Agentes anestésicos & $\begin{array}{l}\text { Anestesia de bajo flujo } \\
\text { Considerar anestesia intravenosa siempre que sea posible } \\
\text { Disminuir o evitar el uso de N2O y Desfluorano }\end{array}$ \\
\hline Dispositivos médicos & $\begin{array}{l}\text { Apagar máquinas y monitores de anestesia cuando no se usen } \\
\text { Utilizar zapatos y gorros individuales, lavables y exclusivos para pabellón } \\
\text { Considerar elementos reusables en pabellón (Laringoscopios, Herramientas quirúrgicas, } \\
\text { máscaras laríngeas, etc.) } \\
\text { Mínimo número de jeringas posible por paciente } \\
\text { No calentar los fluidos de los pacientes, ni aportar calor al paciente si no es estrictamente } \\
\text { necesario } \\
\text { Considerar la frecuencia con que los circuitos anestésicos necesitan lavado }\end{array}$ \\
\hline Iniciativas de sustentabilidad & $\begin{array}{l}\text { Promover el reciclaje en pabellón } \\
\text { Alentar prácticas sustentables }\end{array}$ \\
\hline Reducir & $\begin{array}{l}\text { Formar un comité o equipo multidisciplinario encargado del medioambiente en pabellón } \\
\text { Explorar las posibilidades de disminuir el desperdicio (reducir empaques, utilizar menos } \\
\text { plástico, etc.) }\end{array}$ \\
\hline Reusar & Considerar reutilizar dispositivos pertinentes tras limpieza y esterilización \\
\hline Reciclar & Averiguar acerca de recicladores o plantas de reciclaje locales \\
\hline Investigar & $\begin{array}{l}\text { Averiguar con los fabricanrtes de los equipos el ciclo de vida de sus productos (fármacos } \\
\text { y dispositivos mecánicos o eléctricos) }\end{array}$ \\
\hline
\end{tabular}


Dentro de las limitaciones de nuestro estudio podemos mencionar que no tenemos información detallada, entre otras razones por su diseño, acerca de flujos y concentraciones de gases anestésicos para cada cirugía, por lo tanto, es difícil estimar la HC generada por hora de anestesia en el HBPM, anestesiólogo y/o tipo de cirugía. Por la misma razón es difícil determinar el impacto del uso de técnicas anestésicas alternativas en la generación de $\mathrm{CO} 2 \mathrm{e}$ en nuestro centro.

Taylor señala que "Para llevar a cabo una mejora en la salud planetaria es importante que los sectores que se ven impactados por el cambio climático, como la salud, tomemos un rol proactivo en el entendimiento de nuestro propio impacto ambiental"19. La discusión acerca del efecto de las técnicas anestésicas y las emisiones contaminantes está en desarrollo. Estimar la HC producida por el HBPM es un primer escalón para avanzar en la discusión de que hacemos en las políticas públicas, en los centros clínicos y en particular en la anestesiología para reducir el impacto medioambiental de nuestra actividad.

\section{Referencias}

1. McMichael AJ, Woodruff RE, Hales S. Climate change and human health: present and future risks. Lancet 2006; 367: 859-69.

2. World Health Organization. Climate change and health. 2017. Disponible en: http://www.who.int/mediacentre/ factsheets/fs266/en/ [Consultado el 17 de octubre de 2017].

3.- Sherman J, McGain F. Environmental Sustainability in Anesthesia. Pollution Prevention and Patient Safety. Advances in Anesthesia 2016; 34: 47-61.

4. Ichizawa Y. General Anesthetic Gases and the global Environment. Anesthesia-Analgesia 2011; 112 (1): 213-7.

5. Sustainable Development Unit. NHS Carbon Reduction Strategy Update. 2010. Disponible en: http://www.sduhealth.org.uk/ [Consultado el 17 de octubre de 2017].

6. MacNeill AJ, Lillywhite R, Brown CJ. The impact of surgery on global climate: a carbon Footprint study of operating theatres in three health systems. The Lancet Planetary Health 2017; 1 (9): 381-8.

7. World Resources Institute. World Business Council for Sustainable Development's greenhouse gas protocol: a corporate accounting and reporting standard, revised edition. 2004. Disponible en: http://pdf.wri.org/ ghg_protocol_2004.pdf [Consultado el 17 de octubre de 2017].
8. Secretaría Distrital de Ambiente. Guía para la elaboración del informe de Huella de Carbono Corporativa en entidades públicas del Distrito Capital. 2013. Disponible en: http://ambientebogota.gov.co/en/c/document_library/get_file?uuid $=015755 \mathrm{de}-1 \mathrm{e} 95-49 \mathrm{fb}-8 \mathrm{c} 7 \mathrm{c}-667 \mathrm{c} 4 \mathrm{f}$ b398fa\&groupId=10157 [Consultado el 16 de octubre de 2017].

9. Emission factors in $\mathrm{kg} \mathrm{CO} 2$-equivalent per unit. Canada. 2012. Disponible en: http://www.winnipeg.ca/finance/ findata/matmgt/documents/2012/682-2012/682-2012_ Appendix_H-WSTP_South_End_Plant_Process_Selection_Report/Appendix\%207.pdf [Consultado el 16 de octubre de 2017].

10. Ministry of Environment. Summary of Emissions Factors for the Guidance for Voluntary Corporate Greenhouse Gas. 2015. Disponible en: https://www.mfe.govt. $\mathrm{nz} /$ sites/default/files/media/Climate\%20Change/voluntary-ghg-reporting-summary-tables-emissions-factors-2015.pdf [Consultado el 16 de octubre de 2017].

11. Generalitat de Catalunya. Metodología de inventario de gases de efecto invernadero (GEI), cuestiones básicas. 2011. Disponible en: http://canviclimatic.gencat.cat/ web/.content/home/politiques/inventaris_demissions/ mes_informacio_sobre_metodologies/metodologia_annex_es.pdf [Consultado el 8 de mayo de 2018].

12. Intergovernmental panel on climate change. (s.f.). Glosario.2001. Disponible en: https://www.ipcc.ch/pdf/ glossary/tar-ipcc-terms-sp.pdf [Consultado el 8 de mayo de 2018].

13. Sherman J, Lamers V, Eckelman M. Life Cycle Greenhouse Gas Emissions of Anesthetic Drugs. Anesthesia-analgesia 2012; 114: 1086-90.

14. Sulbaek M, Nielsen O, Wallington T, Karpichev B, Sander S. Assesing the Impact on Global Climate from General Anesthetic Gases. Anesthesia-Analgesia 2012; 114 (5): 1081-5.

15. Ryan S, Nielsen C. Global Warming Potential of Inhaled Anesthetics: Application to Clinical Use. Anesthesia-analgesia 2010; 111 (1): 92-8.

16. Bambarén C, Chú M. Impacto Ambiental del uso del Gas Anestésico Inhalatorio Sevofluorano en un Hospital de Perú. Rev Med Hered 2018; 29 (3): 147-51.

17. Yasny J, White J. Environmental Implications of Anesthetic Gases. Anesth Prog 2012; 59 (4): 154-8.

18. McGain F, Story D, Kayak E, Kashima Y, McAlister S. Workplace Sustaintability: The "Cradle to Grave" View of What We Do. Anesthesia-Analgesia 2012; 114 (5): 1134-9.

19. Taylor T, Mackie P. Carbon footprinting in health systems: one small step. Planetary Health 2017; 1 (9): 357-8. 